\title{
A Data Preprocessing Method Applied to Cluster Analysis on Stock Data by Kmeans
}

\author{
Zhigang Xiong \\ Department of Computer Science and Engineering \\ Shanghai Jiao Tong University \\ Shanghai, China \\ Email:ben.bush@126.com
}

\author{
Assco. Prof. Zhongneng Zhang \\ Department of Computer Science and Engineering \\ Shanghai Jiao Tong University \\ Shanghai, China \\ Email: znzhang@sjtu.edu.cn
}

\begin{abstract}
Recent years, more and more data mining methods are involved in applications like stock price analysis or predication, etc. Kmeans is one commonly used algorithm in those applications. However, those applications only take the technical indices (indicators) as features of data, where may make some important information lost, like the cross of different curves formed by the same technical index with different parameters. In this paper, we propose one way to quantify the variation trend of different curves, which can make kmeans clustering algorithm more effective on stocks analysis.
\end{abstract}

Keywords-clustering; kmens; stock; data process;

\section{INTRODUCTION}

Since the stock market appeared, people have been studying and exploering its inner laws. In these years, there are many analytical methods emerged. Generally, we roughtly divide those analytical methods into two categories, the fundamental analysis and the technical analysis[1]. The fundamental analysis depends on macroeconomic indicators, such as the basic financial status of the company, money flow rate, inflation, etc. And also the fundamental analysis depends on some microeconomic indicators, such as the Listing Corporation's economic behavior and the corresponding economic variables[2]. Then the analyst make a selling or buying decision, after taking all these metrics into consideration. The technical analysis relies on the time series of stock price indices and various technical indicators. However the time series are often formed some complex patterns, which are discontinuous, sltatorial and not convergent. In this field, people usually analyze and make decisions depending on the statistical rules of time series data. Along with the development of data mining, some researchers try to introduce data mining methods into the field of technical analysis.

Clustering is a common method used in stock analysis. Researchers use cluster analysis to obtain the correlationship between different stock index[6], or to determine the period of the stock price[3]. Furthermore some researchers predict the future trend of stock price based on the cluster analysis, such as fuzzy rule based clustering prediction[4]. Among all clustering algorithms, kmeans is the most commonly used clustering algorithm in stock analysis, because the stock data are high dimensions[5], which is a huge challenge for other clustering methods.

However, researchers usually only take the technical indices (indicators) as features of data, which may make the interrelationship between different indices weakened. This means an important information will be lost: the intersection points of the curves formed by the time series of technical indicators. For example RSI (Relative Strength Index) is a famous technical indicator used in stock price trend analysis, which shows the power contrast between buyers and sellers within $\mathrm{n}$ days. Obviously, the 6 days RSI and 12 days RSI are two different time series, will form two different curves. In fact, the intersection points of that two curves may be the perfect deal points. More is people would like to concern the variation of distance between the 6 and 12 days RSI curves.

Hence, in order to solve above problem, we propose a data preprocessing method to quantify the variation of different curves. This proposed method will make the kmeans more effective. In this paper, Section I is an introduction, while in Section II the background knowledge and problem analysis are shown. The detail of our proposed method is placed in Section III. We also take an experiment to examine our proposed method, and its result are in Section IV. In the last section, we make a conclusion.

\section{Background Knowledge \& Problem Analysis}

Cluster analysis are meaningful and useful. It divides data into clusters (groups) where the data share common characteristics. For this reason, cluster analysis make us more aware of the internal relationships of complex systems, like the variation trend of stock price which is a complex system. Some researchers use clustering algorithm to understand discreteness and corelationship of different stock[6]. The different stock in the same cluster indicate that they are very likely to have the same variation trend in the future. Also, clustering methods can be used to predict the stock price, by combing the fuzzy set theory[7]. In general, people are willing to use cluster analysis to guide 
the trading transaction of stocks.

The kmeans clustering technique is simple and commonly used. And kemans is a prototype-based partitional clustering technical which attempts to find the optimal $\mathrm{k}$ partitions. As it is prototype-based, the center of a cluster can be regard as the prototype of all points in such cluster. In other words, the features of a prototype represent the characteristics of all point in such cluster. One merit of kmeans clustering technical is, kmeans can be used for a wide variety of data types. And also kmeans is quite efficient, even though we should run multiple times to obtain a best solution, as it is not a stable algorithm. On the contrary, kmeans may meet a trouble when data contains outliers. However, kmeans is one of the few clustering algorithms that can deal with high dimensional data.

In order to facilitate analysis stock price, scientist create lots of technical indices, like RSI, WR, MA, etc. Generally, a single technical index can not accurately determine the future trend of a stock. In fact, we usually combine several indices to infer the volatility of stock prices. Different parameters of the same technical index also affect our judgement, for example 6 days and 12 days RSI extremely likely do not have the same value on the same day. Thus we should use both 6 and 12 days RSI to analyze the stock price. We can imagine, when using the clustering method to analyze stocks, the features will be a lot (a high dimensions dataset). Hence, kmeans is very suit for stock analysis.

$$
S S E=\sum_{i=1}^{K} \sum_{x \in C_{i}} \operatorname{dist}\left(c_{i}, x\right)^{2}
$$

When evaluate the validity of kmeans, SSE (sum of the squared error) is a good measurement (objective function). Equation 1 shows the defination of SSE, where $C_{i}$ represent cluster $i, c_{i}$ is the center of cluster $i$, dist is the standard Euclidean $\left(L_{2}\right)$ distance between two objects in Euclidean space. Indeed, the SSE represents the entropy of clusters. A smaller SSE means the points are more similiar in the same cluser, and the dataset are well separated by kmeans, where the smaller SSE the better. Thus, it's easily to know that the result with smaller SSE is better. Observing the definition of SSE, we notice that the different features of $x$ are regarded as linear independence, which means the correlationship of different features of $x$ are consider less in this expression. As we have mentioned in above section, the variation trend of two curves formed by the same technical index is the important basis for us to predict the future trend of stock price. In this point of view, a way to quantify the variation trend is desired.

\section{PROPOSED PREPROCESSING METHOD}

In the stock market analysis, most of the technical indicators have parameters. For example, RSI has a parameter $\mathrm{n}$ represent the size of watch window (days), which means we use recent $\mathrm{n}$ days trading data to calculate today's RSI. Usually, we take 12 or 14 as the window size for RSI. However, when conjecture the future trend of stock price, we would like to consider the difference between 6 days RSI and 12 RSI. Looking at the following three examples, each of them represents one real situation.

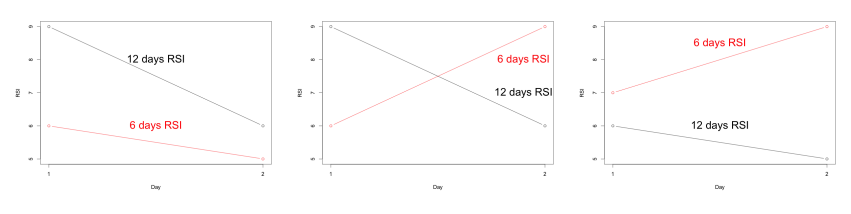

Figure 1. Relations of 6 and 12 days RSI

In Figure 1, the left subfigure shows that 6 days RSI gets close to 12 days RSI on day 2, the middle one shows an intersection is appeared on day 2, while the right subfigure shows that 6 days RSI gets separate on day 2 . As we already know, RSI is used to measure the power contrast between buyers and sellers, the left subfigure of Figure 1 tells us that bayers power is weakening whether in 6 or 12 days, but the speed of decay is slowing down. Similarly, the middle subfigure tells us, the buyer power exceeds the seller on day 2 , and will continue to strengthen. The right subfigure tells us, in recent 6 days, the buyer power significantly stronger than it in 12 days, and the gap is widening. This is a sgin that the buyer confidence is recovering on day 2 .

From the above analysis process we can see, the difference between 6 days RSI and 12 days RSI is quite important. And also, the varation trend of the difference is a key consideration, when analyze the stock data. Hence, we should not only take the time series of RSI as features, but also need to take the difference and the variation trend of difference between 6 and 12 days RSI, when using kmeans to anslyze the stocks. So we add two additional assessments, which can reflect the characteristics of the RSI curve better. The definition is shown below.

$$
\begin{gathered}
\operatorname{Dif}=\operatorname{RSI}(12)-\operatorname{RSI}(6) \\
\operatorname{Trend}(i)=(\operatorname{Dif}(i)-\operatorname{Dif}(i-1)) * \operatorname{sign}(\operatorname{Dif}(i-1))
\end{gathered}
$$

Equation 2 represent the difference between 6 and 12 days RSI. If $\operatorname{Dif}(i)>0$, it means last 12 days buyer power is greate than 6 days on day $i$, more is in recent 6 days, the desire to buy stocks is not strong enough. On the other hand, if $\operatorname{Dif}(i)<0$, it means recently people's desire to buy is more intense. The smaller the Dif(i), the higher the enthusiasm of the purchase, in this case the market may become overbought, and vice versa. 
In Equation 3, the $\operatorname{sign}(x)$ is the sign function. And this equation represent the two RSI curves are close or far away, or cross on day $i$. If $\operatorname{Trend}(i)>0$, it means 6 days RSI gets close to 12 days RSI on day $i$, like the situation in left subfigure of Figure 1 where $\operatorname{Trend}(2)=2$. Specially, when Trend $(i)$ is relatively large and has a different sign with $\operatorname{Dif}(i)$, we can deduce that there is a intersection point occurs on day $i$, and the 6 days RSI curve go through the 12 days RSI curve from bottom to top, like the example in middle subfigure of Figure 1. Otherwise, if Trend $(i)<$ 0 and $\operatorname{Dif}(i)>0$, the 6 days RSI curve go through the 12 days RSI curve frorm top to bottom. In Figure 1, the right subfigure shows the separation of two curves, where the $\operatorname{Trend}(2)=-5<0$.

Table I

TECHNICAL INDICES

\begin{tabular}{|l|l|}
\hline Technical Index & Explanation \\
\hline Open & $\begin{array}{l}\text { The increase rate of today's opening price } \\
\text { relative to the closing price of yesterday }\end{array}$ \\
\hline Close & $\begin{array}{l}\text { The increase rate of today's closing price relative } \\
\text { to today's opening price }\end{array}$ \\
\hline High & $\begin{array}{l}\text { The increase rate of today's highest price relative } \\
\text { to today's opening price }\end{array}$ \\
\hline Volumn & $\begin{array}{l}\text { The increase rate of today's lowest price relative } \\
\text { to today's opening price }\end{array}$ \\
\hline Amount & $\begin{array}{l}\text { The increase rate of today's trading volumn } \\
\text { relative to the volumn of yesterday }\end{array}$ \\
\hline WR & $\begin{array}{l}\text { The increase rate of today's trading amount } \\
\text { relative to the amount of yesterday }\end{array}$ \\
\hline RSI & $\begin{array}{l}\text { A technical analysis oscillator, its goals is try to } \\
\text { high or the, or somewhere else, in recent } \mathrm{n} \text { days }\end{array}$ \\
\hline MA & $\begin{array}{l}\text { It's used to show the power constrast between } \\
\text { buyers and sellers in recent } \mathrm{n} \text { days }\end{array}$ \\
\hline BIAS & $\begin{array}{l}\text { The moving average of stock price of recent } \mathrm{n} \\
\text { days. Here we use its increase rate as the variable }\end{array}$ \\
\hline PSY & $\begin{array}{l}\text { A measure of the degree of the stock price } \\
\text { deviates from the average of n days. The stock } \\
\text { price will tend to return to the average when } \\
\text { BIAS is large }\end{array}$ \\
\hline $\begin{array}{l}\text { Psychological line is a kind of psychological } \\
\text { tendency, which is based on the study of the } \\
\text { investors. To tell us people are willing to buy or } \\
\text { sell the stock in recent n days }\end{array}$ \\
\hline
\end{tabular}

We can see that above two equations can well describe the variation trend of two curves. Thus, we add Dif and Trend as data features, when using kmeans to clustering stock data. In stock analysis, there are many technical indicators (indices) similar to RSI, people are willing to pay attention to the variation trend of two curves formed by different parameters, like WR, BIAS, KDJ and etc. Hence, before doing the cluster analysis, first we calculate the Dif and Trend for those technical indices who require. And then put all Dif and Trend, together with technical indices, as the features of data. We believe that it will have a better result, after doing our proposed data preprocessing method. The date preprocessing flow is shown in Figure 2.

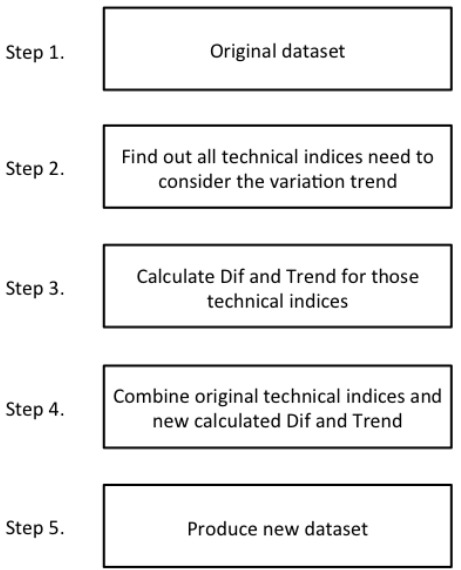

Figure 2. Data Preprocessing Flow

\section{EXPERIMENTAL RESULTS}

We take the time series data of daily CSI 300 index from 2007-01-04 to 2015-06-10 to examine our proposed data preprocessing method. And the technical indices used are shown in Table I. Among those indice, we calculate the Dif and Trend for WR, RSI and BIAS, because people used to pay attention to their variation trend of two curves formed by different parameters.

To demonstrate the effectiveness of our approach, we prepare two datasets, one is the original dataset, and another one is the new dataset with taking the preprocessing method in Figure 2. Table II shows which features are included in which dataset, and the parameters used for each features. The "yes" in column "normal" means we normalize this feature (about the normalization, seeing [8], and the normalization function used is Equation 4.

$$
x[i]=\frac{x[i]-\min (x)}{\max (x)-\min (x)}
$$

In order to observe how our proposed approach affect the results of kmeans, we take $K$ from 2 to 25 , as $K$ is a user specified parameter in kmeans. And as everyone knows, kmeans is not a stable algorithm, the result of each run may be not the same. Thus, for each $K$, we should run serveral times to obtain a good result. In our experiment, for each $K$, we run 30 times.

As we mentioned in Section II, $S S E$ (sum of the squared error) is a good measurement for kmeans, the smaller the $S S E$, the better the result we have. According to Equation 1 , we understand that the more features we have, the larger $S S E$ we may get. Thus, in this case, $S S E$ does not work properly, because serveral extra features are added into the original dataset. To overcome this difficulty, other 
Table II

DATASETS AND PARAMETERS

\begin{tabular}{|l|l|l|l|l|}
\hline & parameter & normal & orig. data & new data \\
\hline open & & yes & yes & yes \\
\hline close & & yes & yes & yes \\
\hline high & & yes & yes & yes \\
\hline low & & yes & yes & yes \\
\hline volumn & & yes & yes & yes \\
\hline amount & & yes & yes & yes \\
\hline wr & 10 & no & yes & yes \\
\hline wr.dif & $\begin{array}{l}\text { wr }(10)- \\
\text { wr }(6)\end{array}$ & no & no & yes \\
\hline wr.trend & & yes & no & yes \\
\hline rsi & 12 & no & yes & yes \\
\hline rsi.dif & $\begin{array}{l}\text { rsi }(12)- \\
\text { si }(6)\end{array}$ & no & no & yes \\
\hline rsi.trend & & yes & no & yes \\
\hline ma & 10 & yes & yes & yes \\
\hline bias & 6 & yes & yes & yes \\
\hline bias.dif & $\begin{array}{l}\text { bias }(12)- \\
\text { bias }(6)\end{array}$ & no & no & yes \\
\hline bias.trend & & yes & no & yes \\
\hline psy & 12 & no & yes & yes \\
\hline & & & \\
\hline
\end{tabular}

measurement criteria should be used. Here we use following assessment to evaluate the results, seeing Equation 5.

$$
\text { SSE.ratio }=\frac{\text { SSE.between }}{\text { SSE.between }+ \text { SSE.within }}
$$

The $S S E$.within in Equation 5 is the $S S E$ which we have mentioned in Section II. And SSE.between is defined as Equation 6, which means the sum of the squared error between points in different clusters. It is not difficult to understand that the higher the $S S E$.between of a clustering, the more separated the clusters are from one another, and the better the result we have. Thus, kmeans has a more validity clustering result, if SSE.ratio is closer to 1, and vice versa.

$$
\text { SSE.between }=\sum_{i=1}^{K} \sum_{x \in C_{i}} \sum_{j \neq i}^{K}\left(c_{j}, x\right)
$$

Using the dataset in Table II, finally, we get the experimental result shown in Figure 3. We see that the curve of $S S E$.ratio obtained by new dataset, which preprocess the data by our proposed method, is significantly over (closer to 1 from) the curve obtained by original dataset. Therefore, we can infer from this result that our approach is feasible and affective, which lead kmeans clustering algorithm to obtain a more volidity partitions.

\section{CONClusion}

There are some defects, if we use kmeans to make a cluster analysis on stock data in traditional way, because usually they only take the technical indices (indicators) as features of data, where some important information may lost, like the cross of different curves formed by the same technical index with different parameters. However, the variation trend of two curves formed by the same technical index with different parameters, including get close, far

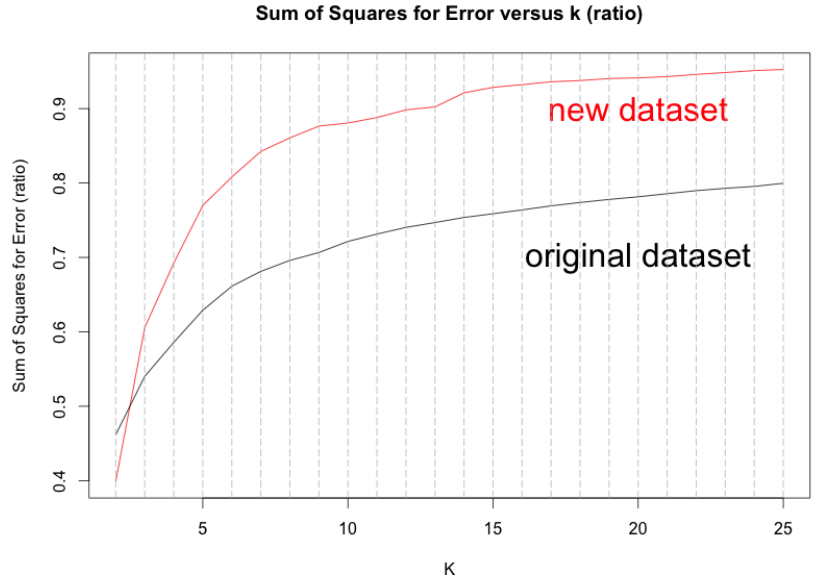

Figure 3. Experimental Result

away and intersection. To compensate for this shortcoming, in this paper, we propose a data preprocessing method to quantify the variation trend, which introduce two extra assessments Dif and Trend, seeing Section II.

After taking an experiment, we observed, after using our proposed preprocessing method, kmeans clustering algorithm will obtain a more effective partitions, which is reflected in a significant increase on SSE.ratio. Therefore, we can say that our method is feasible and effective.

\section{REFERENCES}

[1] Edwards, Robert D., John Magee, and W. H. C. Bassetti. Technical analysis of stock trends. CRC Press, 2007.

[2] Boyer, M. Martin, and Didier Filion. Common and fundamental factors in stock returns of Canadian oil and gas companies. Energy Economics 29.3 (2007): 428-453.

[3] Arnott, Robert D. Cluster analysis and stock price comovement. Financial Analysts Journal 36.6 (1980): 56-62.s

[4] Lai, Robert K., et al. Evolving and clustering fuzzy decision tree for financial time series data forecasting. Expert Systems with Applications 36.2 (2009): 3761-3773.

[5] Donoho, David L. High-dimensional data analysis: The curses and blessings of dimensionality. AMS Math Challenges Lecture (2000): 1-32.

[6] Song, Dong-Ming, et al. Evolution of worldwide stock markets, correlation structure, and correlation-based graphs. Physical Review E 84.2 (2011): 026108.

[7] Liu, Chih-Feng, Chi-Yuan Yeh, and Shie-Jue Lee. Application of type-2 neuro-fuzzy modeling in stock price prediction. Applied Soft Computing 12.4 (2012): 1348-1358.

[8] Visalakshi, N. Karthikeyani, and K. Thangavel. Impact of normalization in distributed $k$-means clustering. international Journal of Soft computing 4.4 (2009): 168-172. 